\title{
Analysis of Selection Schemes for Solving an Optimization Problem in Genetic Algorithm
}

\author{
Poonam Sharma \\ Amity University Haryana \\ M. Tech (Student)
}

\author{
Amit Wadhwa \\ Amity University Haryana \\ Asst. Professor
}

\author{
Komal \\ Amity University Haryana \\ Asst. Professor
}

\begin{abstract}
Genetic algorithm is a population based an adaptive search and optimizations techniques and genetic mimic the natural evolution process. The Genetic operators include selection, crossover and mutation. The aim to present this paper is it gives comparative selection strategies for solving an optimization problem in genetic algorithm and evaluates their performance.
\end{abstract}

\section{Keywords}

Selection, Crossover, Roulette Wheel Selection, Rank Selection, Tournament Selection, Elitism Selection.

\section{INTRODUCTION}

Genetic algorithm is a part of evolutionary algorithm. In genetic algorithm for representation, we can use the fixed length bits strings. [8] In genetic algorithm encoding of Chromosomes are our first step to solve any problem .Genetic algorithm is also dependent on the fitness function. The fitness functions find out the quality of solution such as measurable and returning the fitness value. They perform very well for large scale optimization problem. The main reason to use the genetic algorithm because there are multiple local optima. Selection operator identifies the good solution in a population and makes multiple copies of better string in a new population. [2]It is also called reproduction operator. In reproduction operator we cannot create the new string. In selection method we can randomly pick the chromosome from the population according to evaluation function. If the fitness function is higher the more chance an individual has to be selected. [8]

In crossover operator we can recombine two strings to get a better string. It is also called recombination operator. In which we can create and copy the new string. [4]

In mutation operator we can randomly distributing genetic information. It is used to maintain the diversity in the population. [4]

Further we have been described as: Section 2 describes brief summary of Literature survey. Section 3 describes different types of selection operators. Section 4 gives the description of implementation. Section 5 gives the description of result. Section 6 summarized the conclusion.

\section{LITERATURE SURVEY}

[Anshul Sharma, Anuj Mehta][2] Described a various types of selection strategy in the genetic algorithm procedure to find the optimal solution for the genes and compare the performance in terms of solution quality and find the number of generation for the best solution.

[Tarun Varshney, Aishwary Katiyar, Pankaj Sharma][1] Presented a comparative analysis of selection schemes of genetic algorithm to find an optimum and reliable route in wired networks. Their result to find out the performance and time complexity. Hence observed that the reliable route in wired networks from source node to destination node is successfully found out but the best result gives the rank selection and tournament selection.

[Noraini Mohd Razali, John Geraghty][6] Described three types of selection strategy in the GA procedure to solve TSP and compare their performance in terms of solution quality and number of generations to come out with the best solution. Hence, the rank based roulette wheel can be used for large size problem while tournament selection can be used for small size problem. The best choice is rank selection.

[Chetan Chudasama, S. M. Shah, Mahesh Panchal][7] Described Comparison of Parents Selection Methods of Genetic Algorithm for TSP. Hence their result the elitism selection has generated very fit population as compared to the roulette wheel and tournament selection.

\section{TYPES OF SELECTION OPERATORS \\ 3.1 Roulette Wheel Selection \\ 3.2 Rank Selection \\ 3.3 Tournament Selection \\ 3.4 Elitism Selection}

\subsection{Roulette Wheel Selection}

The role of roulette wheel is the area covered by the entire chromosome in a population as per the fitness value. Every individual gives the slice of roulette wheel and the sizes of slice are directly proportional to the each individual fitness. Now this process is repeated until the desired number of individual is selected. If fitness is higher, the segment size is larger. [2] The fig1: represents the Roulette Wheel 6 individuals with fitness values.

So the $5^{\text {th }}$ individual gives the highest fitness values than any other individual in roulette wheel So that it will chooses the $5^{\text {th }}$ individual than other. It is also called fitness-proportionate selection. 


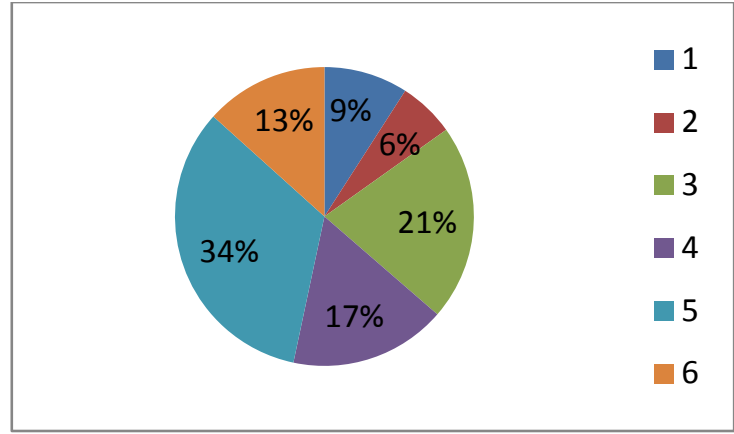

\begin{tabular}{|c|c|}
\hline Population & Fitness \\
\hline 1 & 9.0 \\
\hline 2 & 6.0 \\
\hline 3 & 21.0 \\
\hline 4 & 17.0 \\
\hline 5 & 34.0 \\
\hline 6 & 13.0 \\
\hline
\end{tabular}

Fig 1: Roulette wheel selection

Advantages: It always gives a chance all of them to be selected. [3]

Disadvantages: (a) Roulette wheel selection method cannot be used on minimization problems.

(b) As population converges it loss of selection pressure.

Time complexity: $\mathrm{O}\left(\mathrm{n}^{2}\right)$

\subsection{Rank Selection}

To solve the problem of roulette wheel we use the rank selection. In rank selection first of all rank the population and after that from the ranking every genes receives the fitness. In rank selection we can sort from best to worst. The worst fitness has rank 1, next has rank 2 and the best rank has $\mathrm{N}$, where $\mathrm{N}$ is no. of population. The purpose of rank selection to prevent too quick convergence. [2][8]

Advantages: To prevent too quick convergence.

Disadvantages: populations must be sort on every cycle.

Time complexity: $\mathrm{O}(\mathrm{n} \ln \mathrm{n})+$ time of selection.

\subsection{Tournament Selection}

In tournament selection various tour of a few individual is selected at randomly from population and selected best individual as a parent. We select best fitness values with winner of each tournament. When the tournament size is changed we can easily adjusted selection pressure. [9][10]

\section{Advantages:}

(a) When implementing in parallel it has efficient time complexity.

(b) Fitness scaling or sorting not required.

Disadvantages: This type of selection does not give guarantee to reproduction of best solution. [5]

Time complexity: $\mathrm{O}(\mathrm{n})$

\subsection{Elitism Selection}

In elitism selection we can copy the first best chromosomes or the few best chromosomes to the new population. Firstly, we can arrange the chromosome in the decreasing order. After that selection is applied with every two chromosomes in arrange set. There is no need to any modification we can pass the best individual to next generation. [2][8]

Advantages: This significantly improves the GA's performance because elitism generates very fit population generations.

Time complexity: $\mathrm{O}\left(\mathrm{MN}^{2}\right)$

\section{IMPLEMENTATION}

For implementation, we use the MATLAB tool and De Jong's function 1 . The functionality of real encoding is used to solve the problem of genetic algorithm. In genetic algorithm, the population size has been set 20 and number of generation has been set 10 for best result. Selection and crossover methods are used to find the performance and best result.

\section{RESULTS}

The table 1 shows the chromosome id and their fitness value(x) and after that it shows the graph for chromosome .After applying 10 generation the table 2 show the best result for selection.

Table 1. Chromosome id \& fitness value

\begin{tabular}{|c|c|}
\hline $\begin{array}{c}\text { Chromosome } \\
\text { id }\end{array}$ & Fitness Value \\
\hline 1 & 1.210 \\
\hline 2 & 0.360 \\
\hline 3 & 3.610 \\
\hline 4 & 2.560 \\
\hline 5 & 0.250 \\
\hline 6 & 5.760 \\
\hline 7 & 4.410 \\
\hline 8 & 2.890 \\
\hline 9 & 5.289 \\
\hline 10 & 0.640 \\
\hline 11 & 1.690 \\
\hline
\end{tabular}




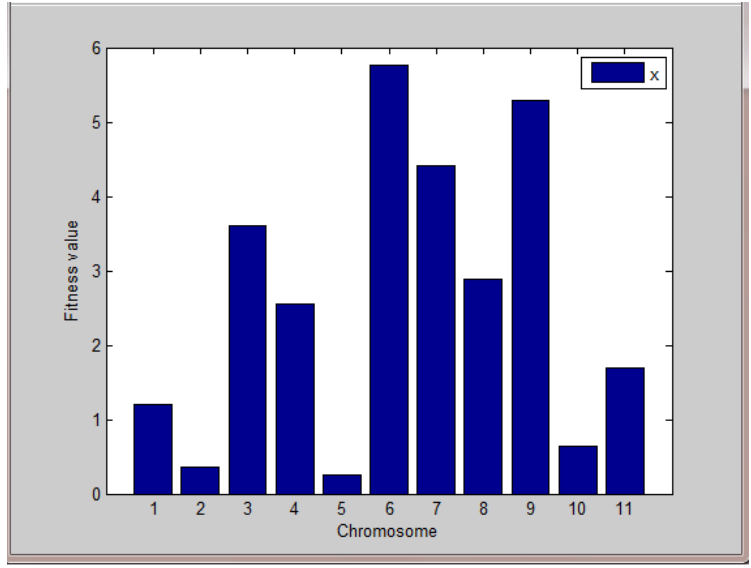

Fig 2: Graph for chromosome

Table 2. After applying 10 generation

\begin{tabular}{|c|c|c|}
\hline Selection & Crossover & After 10 generation \\
\hline Roulette wheel & One point & 1.702 \\
\hline Roulette wheel & Two point & 2.077 \\
\hline Tournament & One point & 1.820 \\
\hline $\begin{array}{c}\text { Tournament } \\
\text { Two point }\end{array}$ & 1.860 \\
\hline $\begin{array}{c}\text { Stochastic } \\
\text { Uniform }\end{array}$ & One point & 1.467 \\
\hline $\begin{array}{c}\text { Stochastic } \\
\text { Uniform }\end{array}$ & Two point & 1.867 \\
\hline
\end{tabular}

We achieve best result when applying roulette wheel selection with two point crossover and tournament selection with one point crossover.

\section{CONCLUSION}

This paper presented the four types of selection operators in the Genetic algorithm. To find out the performance of selection operators and its time complexity for the best result. In Roulette wheel selection the area covered by the entire chromosome in a population as per the fitness value. Now this process is repeated until the desired number of individual is selected. In rank selection first of all rank the population and after that from the ranking every genes receives the fitness. In Tournament Selection We select best fitness values with winner of each tournament. When the tournament size is changed we can easily adjusted selection pressure. In elitism selection we can copy the first best chromosomes or the few best chromosomes to the new population. Hence we achieve best result when applying roulette wheel selection with two point crossover and tournament selection with one point crossover.

\section{REFERENCES}

[1] Tarun Varshney, Aishwary Katiyar, Pankaj Sharma, 2013. "Comparative analysis of selection schemes of genetic algorithm to find an optimum and reliable route in wired networks" , International Journal of Research in Information Technology, Volume-01, Issue-04, April 2013, (238-242).

[2] Anshul Sharma, Anuj Mehta 2013."Review Paper of Various Selection Methods in Genetic Algorithm", International Journal of Advanced Research in Computer Science and Software Engineering Volume03, No-07, July 2013, (1476-1479).

[3] Dr. Rajib Kumar Bhattacharjya, 2013. "Introduction to Genetic Algorithms".

[4] Tom V. Mathew, 2013. "Genetic Algorithm".

[5] Firas Alabsi, Reyadh Naoum, 2012. " Comparison of Selection Methods and Crossover Operations using Steady State Genetic Based Intrusion Detection System", Journal of Emerging Trends in Computing and Information Sciences, Volume-03, No-07, July $2012,1053-1058$

[6] Noraini Mohd Razali, John Geraghty, 2011. "Genetic Algorithm Performance with Different Selection Strategies in Solving TSP", Proceedings of the World Congress on Engineering Volume-02, July 6 - 8, 2011, $1-6$.

[7] Chetan Chudasama, S. M. Shah, Mahesh Panchal, 2011. "Comparison of Parents Selection Methods of Genetic Algorithm for TSP", International Conference on Computer Communication and Networks CSICOMNET, 85-87.

[8] S.N.Sivanandam, S.N.Deepa, 2000. "An Introduction to Genetic Algorithms".

[9] Mitchell Melanie, 1996. “An, Introduction to Genetic Algorithms".

[10] T. Blickle, L. Thiele, 1995. "A Comparison of Selection Schemes used in Genetic Algorithms", TIK-Report, Zurich. 